\title{
Decreased dentin tubules density and reduced thickness of peritubular dentin in hyperbilirubinemia-related green teeth
}

\author{
Rodrigo Neves-Silva ${ }^{1}$, Fabio-Abreu Alves ${ }^{2}$, Alberto Antunes ${ }^{3}$, Mario-Fernando Goes ${ }^{3}$, Marcelo Giannini ${ }^{4}$, \\ Maria-Dânia Tenório ${ }^{5}$, José-Lécio Machado ${ }^{6}$, Adriana-Franco Paes-Leme ${ }^{1,7}$, Marcio-Ajudarte Lopes ${ }^{1}$, Alan- \\ Roger Santos-Silva ${ }^{1}$
}

\author{
${ }^{1}$ Oral Diagnosis Department, Semiology Area, Piracicaba Dental School, University of Campinas (UNICAMP), Piracicaba, Sao \\ Paulo, Brazil \\ ${ }^{2}$ Department of Oral Medicine, A. C. Camargo Cancer Center, Sao Paulo, Brazil \\ ${ }^{3}$ Restorative Dentistry Department, Dental Materials Area, Piracicaba Dental School, University of Campinas (UNICAMP), Pira- \\ cicaba, Sao Paulo, Brazil \\ ${ }^{4}$ Restorative Dentistry Department, Dentistry Area, Piracicaba Dental School, University of Campinas (UNICAMP), Piracicaba, \\ Sao Paulo, Brazil \\ ${ }^{5}$ Pediatric Dentistry Department, School of Dentistry, Federal University of Alagoas (UFAL), Maceio, Alagoas, Brazil \\ ${ }^{6}$ Oral Medicine Department, School of Dentistry, Federal University of Alagoas (UFAL), Maceio, Alagoas, Brazil \\ ${ }^{7}$ Mass Spectrometry Laboratory, Brazilian Biosciences National Laboratory - CNPEM, Campinas, Brazil
}

Correspondence:

Área de Semiologia

Faculdade de Odontologia de Piracicaba - UNICAMP

Av. Limeira, 901, Bairro Areão, Piracicaba

Sao Paulo, Brasil, CEP 13414-903

alanroger@fop.unicamp.br

Received: 21/09/2016

Accepted: $15 / 03 / 2017$

Neves-Silva R, Alves FA, Antunes A, Goes MF, Giannini M, Tenório MD, Machado JL, Paes-Leme AF, Lopes MA, Santos-Silva AR. Decreased dentin tubules density and reduced thickness of peritubular dentin in hyperbilirubinemia-related green teeth. J Clin Exp Dent. 2017;9(5):e622-8. http://www.medicinaoral.com/odo/volumenes/v9i5/jcedv9i5p622.pdf

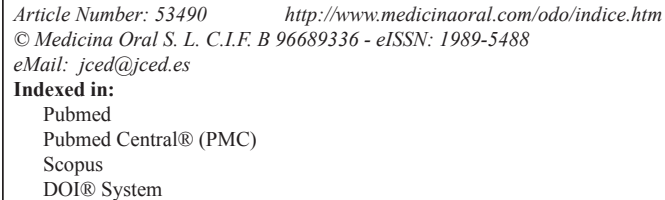

\begin{abstract}
Background: It is stated anecdotally that patients with liver diseases in childhood who develop green teeth have increased risk for rampant caries, which may be secondary to changes in dental structure. The aim of this study was to test the hypothesis that hyperbilirubinemia affects the dentin morphology of green teeth.

Material and Methods: Sixteen primary teeth were prepared and divided into two groups (green teeth, $\mathrm{n}=8$ and control, $\mathrm{n}=8$ ), which were transversely fractured across the cervical third of the dental crowns; dentin was prepared and sputter-coated with gold, and examined under a scanning electron microscope. The mean density and mean diameter of dentin tubules, as well as the thickness of peritubular dentin, were compared.

Results: Hyperbilirubinemia was associated with a decrease in the density of the dentin tubules $(p<.01)$ and the thickness of peritubular dentin of green teeth $(p<.01)$.

Conclusions: There was a correlation between childhood hyperbilirubinemia and changes in the dentin morphology, including a decrease in the density of the dentin tubules and a reduction in the thickness of peritubular dentin in green teeth.
\end{abstract}

Key words: Hyperbilirubinemia, liver disease, childhood, dentin tubules, human teeth, scanning electron microscopy. 


\section{Introduction}

Metabolic diseases, local factors, and systemic factors may affect the color of teeth, causing permanent dental pigmentation (1). Several childhood diseases cause elevated serum levels of bilirubin (hyperbilirubinemia), which accumulate in tissue, such as bones, teeth, skin, and mucosa, and causing them to turn green. Other oral manifestations of hyperbilirubinemia include enamel hypoplasia, dental caries, and retardation of dental and bone development (2-4). The bilirubin accumulates in skin and mucosa only temporarily and is released due to rapid cell turnover; however, the formation of mineralized tissue permanently incorporates the bilirubin. Nonetheless, the pigmentation of the roots of developing teeth stops when the cause of the hyperbilirubinemia is treated, such as with a liver transplant $(3,4)$.

Green teeth pigmentation caused by hyperbilirubinemia is mostly seen in the primary dentition (5-8). However, previous publications have reported green pigmentation in permanent dentition (9-11). Liver diseases, such as biliary atresia (BA) $(3,12)$, hypoplasia of intrahepatic biliary tract (HIHBT) $(13,14)$, and familial cholestasis (FC) (15) are the most commonly associated with green teeth. This study tested the hypothesis that hyperbilirubinemia is able to affect the dentin morphology of green teeth.

\section{Material and Methods}

This research was approved by the local ethical committee for research (protocol number 122/2011).

-Clinicopathological study

Demographic and medical data of patients enrolled in this study regarding gender, age, type of liver disease, liver transplant data and serum levels of bilirubin (direct, indirect, and total) were collected from the patients' charts. Teeth analyzed in this study were obtained from patients who were indicated for tooth extraction prior to receiving a liver transplant at the Oral Medicine Department of AC Camargo Cancer Center, Sao Paulo, Brazil, and the teeth were stored in buffered solution (Fig. 1) (16). Teeth selected for this study did not present disseminated carious lesions or other macroscopic alterations that could compromise the analysis of dentin tissue.

-Mean density and mean diameter of dentin tubules and thickness of peritubular dentin

Sixteen primary green teeth were prepared and divided into two groups. In the group of green teeth, eight teeth were selected: two $(25 \%)$ canines and six $(75 \%)$ incisors. In the control group, eight teeth from healthy children were used: two $(25 \%)$ canines and six $(75 \%)$ incisors. The control teeth were matched with green teeth so that each group had the same amount of teeth in the same anatomical group and also paired by patients' age. All teeth were prepared based on previous studies $(17,18)$

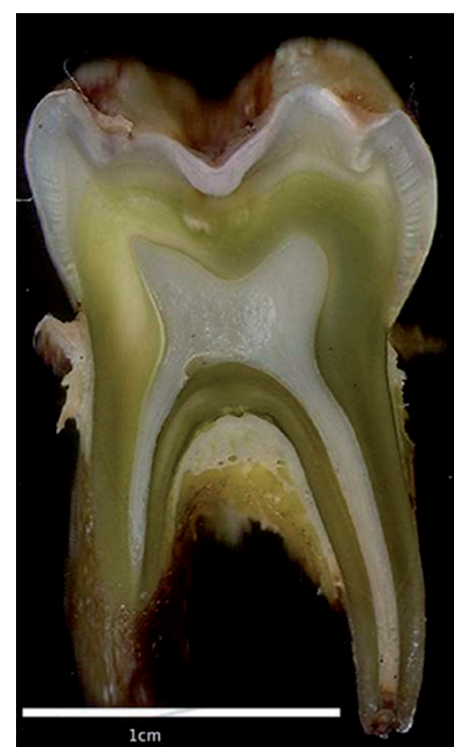

Fig. 1: Macroscopic view of a longitudinal section of a green primary molar from one of the studied patients.

and divided into subgroups. In Group 1, four green teeth were transversely fractured across the cervical third of the crown, and dentin was etched with $37 \%$ phosphoric acid (37 Condac, FGM Dental Products, Joinville, $\mathrm{SC}$, Brazil) for $20 \mathrm{sec}$. In Group 2, four green teeth were transversely fractured across the cervical third of the crown, and dentin was not conditioned. In Group 3, four control teeth were transversely fractured across the cervical third of the crown, and dentin was etched with $37 \%$ phosphoric acid (37 Condac, FGM Dental Products, Joinville, SC, Brazil) for $20 \mathrm{sec}$. In Group 4, four control teeth were transversely fractured across the cervical third of the crown, and dentin was not conditioned.

The specimens were set in stubs with their fractured surface facing up by using colloidal silver adhesive (Electron Microscopy Sciences, Hatfield, PA, USA), sputter-coated with gold (Balzers SCD 050 sputter coater, Balzers Union Aktiengesellschaft, Furstentum Liechtenstein, FL, Germany), and examined in a scanning electron microscope (SEM; JSM-5600 LV - Jeol Ltd., Tokyo, Japan) operating at a voltage of $15 \mathrm{kV}$ and using secondary electrons.

Fifteen SEM micrographs of fractured surfaces of each specimen were randomly obtained for the two groups at a magnification of 2,500x and analyzed in Image $1.45 \mathrm{~s}$, following the methods of previously published articles $(17,19)$.

To determine the tubular density, we counted all the dentinal tubules appearing in each micrograph. For each specimen, we used 15 electron micrographs measuring $175 \mathrm{~mm} \times 114 \mathrm{~mm}$. By using the scale bar appearing in each electron micrograph $(35 \mathrm{~mm}=10 \mu \mathrm{m})$, the dimensions of each micrograph corresponded to 50 and 
$32.57 \mu \mathrm{m}$, thus resulting in an area of $1628.5 \mu \mathrm{m}^{2}$ (= $0.0016285 \mathrm{~mm}^{2}$ ). We divided the number of dentinal tubules present in each electron micrograph by that area $\left(0.0016285 \mathrm{~mm}^{2}\right)$.

To determine the diameter of dentinal tubules, 15 micrographs were obtained from each sample following the same pattern described above and basing our methods on those of Dutra-Correa et al., (17). To take the measurements, we used a scale bar of each micrograph obtained from ImageJ 1.45s. Fifteen dentinal tubules were randomly selected in each micrograph and measured for their horizontal and vertical diameters, totaling 30 measurements per micrograph; therefore, a total of 450 measurements were performed for each specimen. The mean diameter of dentinal tubules of each specimen was then obtained from the average values of the 450 measurements. The mean values were compared among the subgroups (Group 1 versus Group 3 and Group 2 versus Group 4).

To avoid tubule density and diameter variation, we controlled the selection of the tubules by site sampling at the cervical third of the dental crowns and compiling identical dentin fracture levels in all groups. All samples were examined at the same angle of incidence at the SEM, aiming to avoid negative influences during the measurements of the diameter (circular versus ellipsoid diameter). In addition, sampling sites of dentin tubules in all groups were controlled by selection of the tubules to be analyzed.

We established the thickness of peritubular dentin by arriving at the difference between the mean diameter values of fractured-conditioned dentin tubules from the fractured dentin tubules (non-conditioned surface) for green teeth (Group 1 - Group 2) and for control teeth (Group 3 - Group 4). The differences in dentinal tubule diameters between the acid etched and the merely frac- tured specimens revealed an estimate of the thickness of the peritubular dentine "wall" removed in the former samples. All the results were statistically processed using EXCEL ${ }^{\circledR}$ software to obtain average values with their respective standard deviations.

-Statistical analysis

We performed a statistical analysis of the data concerning to investigate the mean density and mean diameter of dentin tubules and the thickness of peritubular dentin for the green and control specimens. A one-way ANOVA parametric nature test was applied using SAS ${ }^{\circledR}$ system with a significance level of $5 \%(\alpha=.05)$.

\section{Results}

-Clinicopathological study

The ages of the eight patients enrolled in this study ranged from 8 to 12 years with a mean age of 10 years; four were male and four were female. All patients had been diagnosed with hyperbilirubinemia in childhood; four had BA, one had primary biliary cirrhosis (PBC), one had HIHBT, one had FC, and one had liver cirrhosis (LC). Clinicopathological data are presented in table 1. -Mean density and diameter of dentin tubules and thickness of peritubular dentin

In transversely fractured green and normal teeth, the average values of mean density of the dentin tubules were obtained from measurements registered in each micrograph. The measures concerning the mean density of the dentin tubules varied from 15.1 to 20.6 (tubules $/ \mathrm{mm}^{2}$ ) to groups I and II, respectively. The results revealed the following averages of the mean density of the dentin tubules to groups III and IV: 34.2 to 29.1 (tubules $/ \mathrm{mm}^{2}$ ), respectively (Table 2 ). The recorded average values of the mean diameter of the dentin tubules were $0.95 \mu \mathrm{m}$ (group I), $0.78 \mu \mathrm{m}$ (group II), $1.09 \mu \mathrm{m}$ (group III), and $0.73 \mu \mathrm{m}$ (group IV), (Table 3 ). The authors also verified

Table 1: Clinical features of patients.

\begin{tabular}{|c|c|c|c|}
\hline Patient & Age (year) & Gender & Underlying disease \\
\hline 1 & 11 & $\mathrm{M}$ & Biliary atresia \\
\hline 2 & 12 & $\mathrm{M}$ & Hypoplasia of intrahepatic biliary tract \\
\hline 3 & 12 & $\mathrm{M}$ & Familial cholestasis \\
\hline 4 & 8 & $\mathrm{~F}$ & Biliary atresia \\
\hline 5 & 10 & $\mathrm{~F}$ & Primary biliary cirrhosis \\
\hline 6 & 10 & $\mathrm{~F}$ & Biliary atresia \\
\hline 7 & 11 & $\mathrm{M}$ & Liver cirrhosis \\
\hline 8 & 11 & $\mathrm{~F}$ & \\
\hline
\end{tabular}

M: male. F: female. 
Table 2: Mean density of the dentin tubules (tubules $/ \mathrm{mm}^{2}$ ).

\begin{tabular}{|c|c|c|c|}
\hline \multicolumn{2}{|c|}{ Group 1} & Group 3 & $p$ value \\
\hline Specimens & Density $\mathrm{mm}^{2}$ & Density $\mathrm{mm}^{2}$ & .0020 \\
\hline 1 & $17.3 \pm 8,0$ & $40.4 \pm 12.1$ & \\
\hline 2 & $17.3 \pm 3,3$ & $39.8 \pm 5.9$ & \\
\hline 3 & $11.7 \pm 3,2$ & $28.1 \pm 3.5$ & \\
\hline 4 & $14.1 \pm 3,0$ & $28.6 \pm 4.3$ & \\
\hline Mean & 15.1 & 34.2 & \\
\hline \multicolumn{2}{|c|}{ Group 2} & Group 4 & $p$ value \\
\hline Specimens & Density $\mathrm{mm}^{2}$ & Density $\mathrm{mm}^{2}$ & .2674 \\
\hline 1 & $20.6 \pm 2.8$ & $30.7 \pm 8.9$ & \\
\hline 2 & $30.8 \pm 6.0$ & $20.0 \pm 3.8$ & \\
\hline 3 & $11.9 \pm 1.2$ & $45.0 \pm 21.8$ & \\
\hline 4 & $18.9 \pm 7.1$ & $20.8 \pm 6.1$ & \\
\hline Mean & 20.6 & 29.1 & \\
\hline
\end{tabular}

Group 1: etched green teeth. Group 2: non-etched green teeth. Group 3: etched control teeth. Group 4: non-etched control teeth.

Table 3: Mean diameter of dentin tubules.

\begin{tabular}{|c|c|c|c|}
\hline \multicolumn{2}{|c|}{ Group 1} & Group 3 & $p$ value \\
\hline Specimens & Mean diameter $(\mu \mathrm{m})$ & Mean diameter $(\mu \mathrm{m})$ & .1285 \\
\hline 1 & $0.91 \pm 0.22$ & $1.02 \pm 0.13$ & \\
\hline 2 & $1.16 \pm 0.09$ & $1.12 \pm 0.09$ & \\
\hline 3 & $0.85 \pm 0.11$ & $1.17 \pm 0.06$ & \\
\hline 4 & $0.89 \pm 0.14$ & $1.05 \pm 0.16$ & \\
\hline Mean & 0.95 & 1.09 & \\
\hline \multicolumn{2}{|c|}{ Group 2} & Group 4 & $p$ value \\
\hline Specimens & Mean diameter $(\mu \mathrm{m})$ & Mean diameter $(\mu \mathrm{m})$ & .6189 \\
\hline 1 & $0.78 \pm 0.08$ & $0.77 \pm 0.11$ & \\
\hline 2 & $0.99 \pm 0.17$ & $0.79 \pm 0.08$ & \\
\hline 3 & $0.70 \pm 0.03$ & $0.74 \pm 0.08$ & \\
\hline 4 & $0.65 \pm 0.07$ & $0.65 \pm 0.08$ & \\
\hline Mean & 0.78 & 0.73 & \\
\hline
\end{tabular}

Group 1: etched green teeth. Group 2: non-etched green teeth. Group 3: etched control teeth Group 4: non-etched control teeth.

that there was a reduction in the thickness of peritubular dentin in green teeth group when compared to control group. The measures of the thickness of peritubular dentin varied from 0.13 to $0.24 \mu \mathrm{m}$ in green teeth group, and varied from 0.25 to $0.42 \mu \mathrm{m}$ in the control group (Table 4). During the semi-quantitative analysis of peritubular dentin, there was no evidence of morphological changes or variations in its structural features (Figs. 2,3).

-Statistical analysis

The mean density of dentin tubules showed the greatest significant difference between Groups 1 and $3(p<.01)$; however, the control group showed the highest density. The thickness of peritubular dentin in green teeth showed significant differences between the mean values for Groups 1 and 2 and Groups 3 and $4(p<.01)$. Control teeth presented the highest mean thickness. The statistical analysis of the presence of hyperbilirubinemia on the mean diameter of dentin tubules compared between Groups 1 and 3, and on the mean diameter of dentin tubules compared between Groups 2 and 4 did not show differences $(p>.05)$. 
Table 4: Thickness of peritubular dentin.

\begin{tabular}{|c|c|c|c|}
\hline & \multicolumn{2}{|c|}{ Thickness $(\mu \mathrm{m})$} & $p$ value \\
\hline \multicolumn{4}{|l|}{ Specimens of green teeth } \\
\hline 1 & Group 1- Group 2 & 0.13 & .0084 \\
\hline 2 & Group 1- Group 2 & 0.17 & \\
\hline 3 & Group 1- Group 2 & 0.15 & \\
\hline \multirow[t]{2}{*}{4} & Group 1- Group 2 & 0.24 & \\
\hline & Standard deviation & 0.04 & \\
\hline Specimens of control teeth & \multicolumn{2}{|c|}{ Thickness $(\mu \mathrm{m})$} & \\
\hline 1 & Group 3 - Group 4 & 0.25 & \\
\hline 2 & Group 3 - Group 4 & 0.33 & \\
\hline 3 & Group 3 - Group 4 & 0.42 & \\
\hline \multirow[t]{2}{*}{4} & Group 3 - Group 4 & 0.40 & \\
\hline & Standard deviation & 0.08 & \\
\hline
\end{tabular}

Group 1: etched green teeth. Group 2: non-etched green teeth. Group 3: etched control teeth. Group 4: non-etched control teeth.
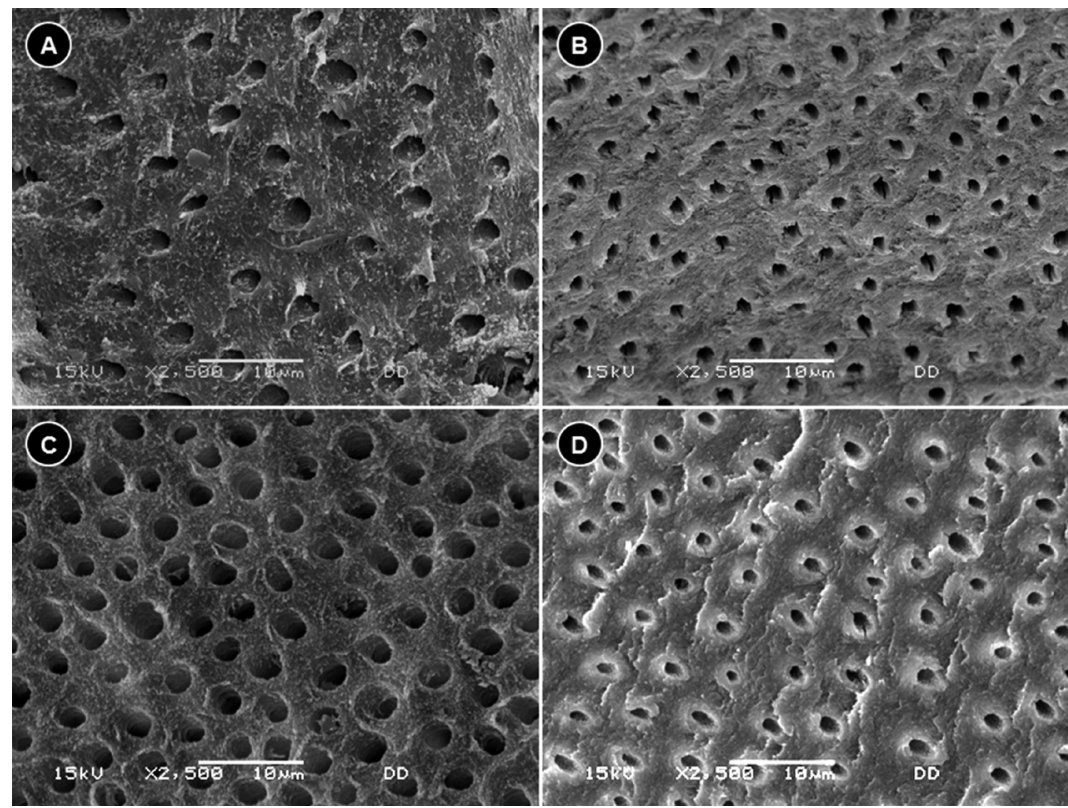

Fig. 2: Density and diameter of the dentin tubules by SEM. A. Dentin tubules of Group 1. B Dentin tubules of Group 2. C. Dentin tubules of Group 3. D. Dentin tubules of Group 4. SEM: scanning electron microscopy.

\section{Discussion}

The current analyses demonstrated that hyperbilirubinemia led to a decrease in the density of the dentin tubules in the green teeth. Remarkably, the values found in the present study are similar to those previously described for normal human dentin both in permanent (20-23) and primary (24) anterior teeth; the number of tubules varied from 3.28 to 32.31 per square millimeter in such studies. Another study (25) compared tubule density between primary and permanent molars and suggested that tubule density was greater in primary molars (primary:
124.32/ $/ \mathrm{mm}^{2}$ versus permanent: $45.97 / \mathrm{mm}^{2}$ ). Interestingly, this is the first study suggesting that high levels of bilirubinemia could yield a dentin with fewer tubules (26-28). The comprehension of the pathophysiological basis of the dentin changes secondary to hyperbilirubinemia is beyond the limits of the current study, but these data certainly open new avenues of potential research and therapy.

Conversely, hyperbilirubinemia did not alter the diameter of dentin tubules in green teeth. The studies examining permanent (20-23) and primary (24) teeth observed 


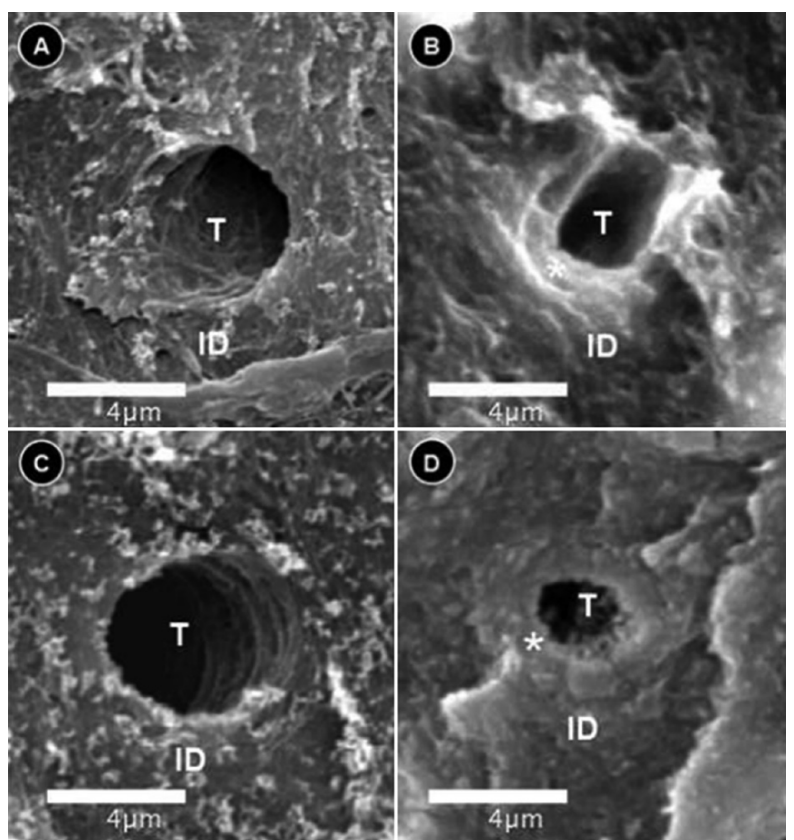

Fig. 3: Peritubular dentin by SEM. A. Dentin tubule of Group 1. B. Dentin tubule of Group 2. C. Dentin tubule of Group 3. D. Dentin tubule of Group 4. ID: intertubular dentin, * = peritubular dentin, T: dentin tubule. SEM: scanning electron microscopy.

values ranging from 0.50 to $1.94 \mu \mathrm{m}$. It was showed that comparing the incisal, middle and cervical thirds, no statistically significant differences were observed in relation to diameter of coronal dentinal tubules of non-erupted deciduous incisors (24), based on results as showed previous, this study only evaluated dentin tubules of cervical third, which presented dentin-tubules measures similar to findings of Costa et al., 2002 (24). Another study analyzed the diameter of dentin tubules in primary molars and found that tubules' diameters ranged from 0.96 to $1.29 \mu \mathrm{m}$, suggesting that different anatomic groups of teeth may present similar values for the diameter of the tubules (25). The findings of lack of altered diameter of dentin tubules in this study represent an interesting perspective for treatment of patients who developed hyperbilirubinemia in childhood because it is known that the diameter of dentin tubules influence dental adhesion, since they are related to dentinal permeability, and the smaller diameter may account for the lower permeability, that is because the efficacy of most of the current adhesives depends on the infiltration of the resin into the dentin $(22,24)$.

The control group of normal teeth specimens showed peritubular dentin with approximately twice the value of the thickness of peritubular dentine in green teeth (apparently, this is the first study to determine the thickness of peritubular dentine in primary teeth). This suggests that hyperbilirubinemia was able to reduce the thickness of peritubular dentin of green teeth probably affecting the physiology of odontoblasts because each odontoblast secrete additional noncollagenous components from the end of its cell process and this matrix mineralizes rapidly between the previously formed intertubular dentine and the odontoblast process, and constitutes the peritubular dentin, containing mostly apatite crystals with little organic matrix $(29,30)$. In the context of the current study that was based on deciduous teeth samples, dentin changes related to hyperbilirubinemia certainly occurred during dentinogenesis, which was probably due to its negative impact on odontoblasts metabolism during dentin formation, a biological event previously suggested by several authors $(2,3,5,6,26-29,31)$.

The observed lower densities of dentin tubules as well as the reduction of the thickness of peritubular dentine in green teeth may be a consequence of long-standing childhood hyperbilirubinemia, which may be able to alter the patterns of dentin mineralization during odontogenesis $(3,4)$. The findings of this study highlighted the importance of tooth structure to dental treatment because bond strength used for evaluations of adhesive material performance are influenced by structural characteristics such as diameter and the number of dentinal tubules per $\mathrm{mm}^{2}$, as well as the relative amount of peritubular and intertubular dentin $(25,30)$.

Based on the findings of the current study, it is possible that hyperbilirubinemia accounts for changes in dentin. It is also important to consider possible toxic effects of liver failure and $\mathrm{PBC}$ on teeth development and consequent dentin changes; however, none of the studied patients presented clinical evidence of retarded growth. The changes in the dentin of hyperbilirubinemia-related green teeth described herein seem to result from a compromised process of dentin mineralization. However, further studies are necessary to explain the involved mechanisms. Besides, a study based on teeth of affected patients after liver transplantation would be helpful to determine if normal bilirubin levels restore the function of the dentin-pulp complex and lead to a normal pattern of dentin tubules and peritubular dentin. This study supported the tested hypothesis that hyperbilirubinemia would be able to alter the morphology of dentin, and concluded that different liver diseases of childhood can alter the dentin morphology of green teeth, which is a secondary indicator for hyperbilirubinemia.

\section{References}

1. Watts A, Addy M. Tooth discolouration and staining: a review of the literature. Br Dent J. 2001;190:309-16.

2. Shibata T, Watanabe K, Oda H, Arisue M, Kurosawa T, Tohma M, et al. Experimental bilirubin pigmentation of rat dentine and its detection by a qualitative analytical method. Arch Oral Biol. 1996;41:509-11.

3. Sommer S, Magagnin K, Kramer PF, Tovo MF, Bervian J. Green teeth associated with neonatal hyperbilirubinemia caused by biliary atresia: review and case report. J Clin Pediatr Dent. 2010;35:199-202. 4. Fernandes KS, Magalhaes M, Ortega KL. Green teeth. J Pediatr. 2011;158:510.

5. Guimaraes LP, Silva TA. Green teeth associated with cholestasis caused by sepsis: a case report and review of the literature. Oral Surg Oral Med Oral Pathol Oral Radiol Endod. 2003;95:446-51. 
6. Alto LA, Pomarico L, Souza IP, Janini ME. Green pigmentation of deciduous teeth: report of two cases. J Dent Child. 2004;71:17982.

7. Guadagni MG, Cocchi S, Tagariello T, Piana G. Case report: Alagi1le syndrome. Minerva Stomatol. 2005;54:593-600.

8. Tjon ATWE, Houwen RH. Green teeth. Arch Dis Child 2007:92:250

9. Stewart DJ. A polychromatic specimen. The result of jaundice and tetracycline. Dent Pract Dent Rec. 1967;17:313-4.

10. Morisaki I, Abe K, Tong LS, Kato K, Sobue S. Dental findings of children with biliary atresia: report of seven cases. ASDC J Dent Child. 1990;57:220-3.

11. Zaia AA, Graner E, de Almeida OP, Scully C. Oral changes associated with biliary atresia and liver transplantation. J Clin Pediatr Dent. 1993;18:38-42.

12. Belanger GK, Sanger R, Casamassimo PS, Bystrom EB. Oral and systemic findings in biliary atresia: report of 11 cases. Pediatr Dent. 1982;4:322-26.

13. Alagille D. Management of paucity of interlobular bile ducts. J Hepatol. 1985;1:561-5.

14. De Tommaso AM, Kawasaki AS, Hessel G. Paucity of intrahepatic bile ducts in infancy-experience of a tertiary center. Arq Gastroenterol. 2004;41:190-2.

15. Balistreri WF, Bezerra JA, Jansen P, Karpen SJ, Shneider BL, Suchy FJ. Intrahepatic cholestasis: summary of an American Association for the Study of Liver Diseases single-topic conference. Hepatology. 2005;42:222-35.

16. Silva AR, Alves FA, Antunes A, Goes MF, Lopes MA. Patterns of demineralization and dentin reactions in radiation-related caries. $\mathrm{Ca}-$ ries Res. 2009;43:43-9.

17. Dutra-Correa M, Anauate-Netto C, Arana-Chavez VE. Density and diameter of dentinal tubules in etched and non-etched bovine dentine examined by scanning electron microscopy. Arch Oral Biol. 2007;52:850-5.

18. Kuwajima K, Ajisaka M, Oyanagi T, Kodama T, Aoki K. Scanning electron microscope study of Tomes' fibers. J Nihon Univ Sch Dent. 1970;12:112-5.

19. Schneider CA, Rasband WS, Eliceiri KW. NIH Image to ImageJ: 25 years of image analysis. Nature methods. 2012;9:671-5.

20. Mjor IA, Nordahl I. The density and branching of dentinal tubules in human teeth. Arch Oral Biol. 1996;41:401-12.

21. Garberoglio R, Brannstrom M. Scanning electron microscopic investigation of human dentinal tubules. Arch Oral Biol. 1976;21:35562.

22. Koutsi V, Noonan RG, Horner JA, Simpson MD, Matthews WG, Pashley DH. The effect of dentin depth on the permeability and ultrastructure of primary molars. Pediatr Dent. 1994;16:29-35.

23. Komabayashi T, Nonomura G, Watanabe LG, Marshall Jr GW, Marshall SJ. Dentin tubule numerical density variations below the CEJ. J Dent. 2008;36:953-8.

24. Costa LR, Watanabe IS, Kronka MC. Coronal dentinal tubules of non-erupted deciduous incisors. Brazilian oral research. 2002;16:12-7.

25. Lenzi TL, Guglielmi CAB, Arana-Chavez VE, Raggio DP. Tubule density and diameter in coronal dentin from primary and permanent human teeth. Microsc Microanal. 2013;19:1445-9.

26. Hals E, Nielsen K. Dental changes in experimental bile duct obstruction in rodents. Arch Oral Biol. 1969;14:151-60.

27. Watanabe K, Shibata T, Kurosawa T, Morisaki I, Kinehara M, Igarashi S, et al. Bilirubin pigmentation of human teeth caused by hyperbilirubinemia. J Oral Pathol Med. 1999;28:128-30.

28. Walsha LJ, Liub JY, Verheyen P. Tooth Discolouration and Its Treatment Using KTP Laser-assisted Tooth Whitening. JOLA. 2004:4:7-21.

29. Arana-Chavez VE, Massa LF. Odontoblasts: the cells forming and maintaining dentine. Int J Biochem Cell Biol. 2004;36:1367-73.

30. Marshall Jr. GW, Marshall SJ, Kinney JH, Balooch M. The dentin substrate: structure and properties related to bonding. J Dent. 1997;25:441-58.
31. Carrillo A, Rezende KM, de Carrillo SR, Arana-Chavez VE, Bonecker M. Hyperbilirubinemia and intrinsic pigmentation in primary teeth: a case report and histological findings. Pediatr Dev Pathol. 2011;14:155-6.

\section{Acknowledgements}

We thank Adriano Luis Martins from the Pathology Area, Piracicaba Dental School, for his contribution during SEM examination. The Human Teeth Bank (BDH) from the School of Dentistry of Federal University of Alagoas (FO-UFAL), Brazil, donated the control teeth. The authors would like to acknowledge The National Council for Scientific and Technological Development (CNPq, Brazil, processes 474697/2012-6 and 122167/2012-0), Sao Paulo Research Foundation (FAPESP, Brazil, process 2013/18402-8), Alagoas Research Foundation (FAPEAL - process number: 20051131340-2), and The Coordination for Specialization of Higher Level Education People (CAPES, Brazil, process PROEX 758/2012) for supporting this research.

\section{Conflict of Interest}

The authors have declared that no conflict of interest exist. 